\title{
Research on Risk Assessment of Photovoltaic Building Project by Triangular Fuzzy Number Method
}

\author{
Yi Liu * \\ School of Economics and Management \\ North China Electric Power University \\ Beijing, China \\ davisliuyi@126.com \\ *Corresponding author \\ Qingzhe Meng \\ School of Economics and Management \\ North China Electric Power University \\ Beijing, China \\ saulzhe@163.com \\ 86-10-61773094
}

\author{
Xintong Dai \\ School of Economics and Management \\ North China Electric Power University \\ Beijing, China \\ 317088683@qq.com
}

\begin{abstract}
Conventional energy shortage and the increasing demand for energy have made energy saving a global-concerned problem. Building integrated photovoltaic project has become an important way to relieve the energy and demand contradiction. Recently, the development of photovoltaic building project in china is immature, with many kind of risk. This paper firstly builds a risk assessment model of photovoltaic building project based on triangular fuzzy number method. Then it does an example research according to a real project which risk is medium.
\end{abstract}

Keywords- photovoltaic building; risk assessment; triangular fuzzy number

\section{INTRODUCTION}

Conventional energy shortage and the increasing demand for energy have made energy saving a global-concerned problem. Accelerating the development and utilization of renewable energy becomes an important way to relieve the energy and demand contradiction. Photovoltaic building has been adopted and popularized widely at home and abroad, as energy saving project combines renewable energy and building energy efficiency perfectly[1]. As a new kind of energy utilization technology which integrates solar energy and building organically, photovoltaic building project, namely building integrated photovoltaic project, is a new building form that makes it a part of the building by organically binding the solar power system(photovoltaic power generation matrix)to the building, and then providing the building or accessing grid with processed electric energy generated by generating device[2]."Medium-long Term Plan of Renewable Energy” of china clearly puts forward the acceleration of the popularization and application of building integrated photovoltaic project, integrated rooftop solar energy grid- connected photovoltaic generation device.20,000 rooftop photovoltaic generation projects will be built up in china by the year of 2020 and the total volume will be 1000,000 KW[3].

Although photovoltaic building project has advantages of nice environmental benefits, renewability, out of exhaustion, short capital construction period, flexible install capacity and so on[4],we must realize that photovoltaic building development technology in china at present is still immature and many risks will be encountered during scheme design, construction and even project management. Risk analysis and evasion will directly affect the photovoltaic building project and even the performance of the enterprise, thus, the core problem of risks analysis and evasion of photovoltaic building project is further discussing risk factors, which have an impact on photovoltaic building project[5].This paper analyzes the risks of photovoltaic building from the aspects of natural environment, technology, economic, policy in detail, establishes risks evaluation system, researches risks evaluation application research with triangular fuzzy numbers, puts forward control scheme of photovoltaic building integrated risk based on risks analysis result.

\section{RISK EVALUATION INDEX SYSTEM}

Because of the variety of risk influence factors of the photovoltaic building project, comprehensive analysis of photovoltaic building project risks from different dimensions and different administrative levels is needed. In this paper, we will establish the risk evaluation index system of photovoltaic building project from four dimensions of natural environment, technology, economic and policy, evaluate the whole photovoltaic building project by introducing triangular fuzzy 
numbers. Risk evaluation index system of photovoltaic building project is showed in Table I.

TABLE I. COMPREHENSIVE RISK EVALUATION INDEX SYSTEM

\begin{tabular}{|c|c|c|}
\hline risk & first-level factors & indexes \\
\hline \multirow{6}{*}{$\begin{array}{c}\text { natural } \\
\text { environment } \\
\text { risks(N) }\end{array}$} & \multirow{4}{*}{$\begin{array}{l}\text { geographical } \\
\text { environment and } \\
\text { meteorological } \\
\text { indexes(N1) }\end{array}$} & latitude and longitude(N11) \\
\hline & & sunshine duration(N12 \\
\hline & & altitude and pressure(N13) \\
\hline & & temperature and humidity(N14) \\
\hline & \multirow{2}{*}{$\begin{array}{l}\text { extreme environment } \\
\text { index(N2) }\end{array}$} & geological hazardindex(N21) \\
\hline & & comprehensive extreme climate (N22) \\
\hline \multirow{22}{*}{$\begin{array}{l}\text { technology } \\
\text { risks(T) }\end{array}$} & \multirow{5}{*}{$\begin{array}{l}\text { echnical scheme } \\
\text { designrisk(T1) }\end{array}$} & technology design feasibility(T11) \\
\hline & & commission design qualification(T12) \\
\hline & & information and data authenticity(T13) \\
\hline & & design innovation(T14) \\
\hline & & designapplicability(T15) \\
\hline & \multirow{3}{*}{$\begin{array}{l}\text { equipment } \\
\text { selectionrisk(T2) }\end{array}$} & equipment security(T21) \\
\hline & & equipment stability(T22) \\
\hline & & $\begin{array}{lll}\text { equipment } & \text { performance } & \text { price } \\
\text { ratio(T23) }\end{array}$ \\
\hline & \multirow{4}{*}{$\begin{array}{l}\text { personnel } \\
\text { construction safety } \\
\text { risk(T3) }\end{array}$} & constructors' quality(T31) \\
\hline & & personnel safety facility(T32) \\
\hline & & training and supervision (T33) \\
\hline & & construction equipment security(T34) \\
\hline & \multirow{3}{*}{\begin{tabular}{lr}
\multicolumn{2}{l}{ equipment } \\
and & debugtlation \\
risk(T4) & \\
\end{tabular}} & job specification soundness(T41) \\
\hline & & operators' relative qualification(T42) \\
\hline & & technology support fault(T43) \\
\hline & \multirow{3}{*}{$\begin{array}{l}\text { construction period } \\
\text { management } \\
\text { dispatchrisk(T5) }\end{array}$} & project management team(T51) \\
\hline & & management dispatch scheme(T52) \\
\hline & & information condition(T53) \\
\hline & $\begin{array}{ll}\begin{array}{l}\text { relay } \\
\text { risk(T6) }\end{array} & \text { protection } \\
\end{array}$ & relay protection risk(T61) \\
\hline & $\begin{array}{ll}\text { power supply } \\
\text { reliabilityrisk(T7) }\end{array}$ & power reliability risk(T71) \\
\hline & $\begin{array}{ll}\begin{array}{l}\text { power } \\
\text { risk(T8) }\end{array} & \text { stability } \\
\end{array}$ & power stability risk(T81) \\
\hline & power quality risk(T9) & power quality risk(T91) \\
\hline \multirow{15}{*}{$\begin{array}{l}\text { economic } \\
\operatorname{risks}(E)\end{array}$} & $\begin{array}{ll}\text { pool } & \text { purchase } \\
\text { price(E1) } & \\
\end{array}$ & pool purchase price(E11) \\
\hline & $\begin{array}{l}\text { electricity to access } \\
\text { grid(E2) }\end{array}$ & electricity to access grid(E21) \\
\hline & $\begin{array}{ll}\text { operation } & \text { cost } \\
\text { risk(E3) } & \\
\end{array}$ & operation cost risk(E31) \\
\hline & extra cost risk(E4) & extra cost risk(E41) \\
\hline & efficiency risk(E5) & efficiency risk(E51) \\
\hline & \multirow{3}{*}{ profit ability(E6) } & operating profit ratio(E61) \\
\hline & & operating gross profit rate(E62) \\
\hline & & the profit margin of costs(E63) \\
\hline & \multirow{3}{*}{$\begin{array}{l}\text { debt } \quad \text { paying } \\
\text { ability(E7) }\end{array}$} & cash to current liability ratio(E71) \\
\hline & & equity ratio(E72) \\
\hline & & obtained interest multiple(E73) \\
\hline & \multirow{2}{*}{$\begin{array}{l}\text { development } \\
\text { ability(E8) }\end{array}$} & income growth rate(E81) \\
\hline & & net profit growth rate(E82) \\
\hline & \multirow{2}{*}{ operation ability(E9) } & accounts receivable turnover ratio(E91) \\
\hline & & turnover rate of fixed assets(E92) \\
\hline \multirow{5}{*}{$\begin{array}{l}\text { policy } \\
\text { risks(P) }\end{array}$} & \multirow{2}{*}{ industrial policy(P1) } & investment allowance(P11) \\
\hline & & offset by tax revenue(P12) \\
\hline & grid policy(P2) & purchase electricity compensation(P21) \\
\hline & continuity(P3) & continuity index(P31) \\
\hline & operability(P4) & operability index(P41) \\
\hline
\end{tabular}

2) Classify the photovoltaic building project risk set Y.Let be the classification of Y,denoted by ,that is called the secondlevel factors set.

3) Determine index weight of various photovoltaic building project risk evaluation indexes with the method of Fuzzy-AHP. We get the judgment matrix with comparison of indexes under one criterion and the matrix is:

$$
\tilde{a}=\left[\begin{array}{cccc}
{[1,1,1]} & {\left[a_{12}^{L}, a_{12}^{M}, a_{12}^{U}\right]} & \cdots & {\left[a_{1 n}^{L}, a_{1 n}^{M}, a_{1 n}^{U}\right]} \\
{\left[a_{21}^{L}, a_{21}^{M}, a_{21}^{U}\right]} & {[1,1,1]} & \cdots & {\left[a_{2 n}^{L}, a_{2 n}^{M}, a_{2 n}^{U}\right]} \\
\cdots & \cdots & \ddots & \cdots \\
{\left[a_{n 1}^{L}, a_{n 1}^{M}, a_{n 1}^{U}\right]} & {\left[a_{n 2}^{L}, a_{n 2}^{M}, a_{n 2}^{U}\right]} & \cdots & {\left[a_{n n}^{L}, a_{n n}^{M}, a_{n n}^{U}\right]}
\end{array}\right]
$$

Among them, $\left[a_{i j}^{L}, a_{i j}^{M}, a_{i j}^{U}\right]_{\text {represents the importance of the }}$ ith and the jth in the proportion by means of triangular fuzzy numbers,and its value is expressed with triangular fuzzy numbers reciprocal scale showed in Table II.

TABLE II. UDGMENT MATRIX SCALE

\begin{tabular}{|l|l|}
\hline \multicolumn{1}{|c|}{ criticality class } & \multicolumn{1}{c|}{$a_{i j}$ scale } \\
\hline i and $j$ are equally important & $\tilde{1}=[1,1,2]$ \\
\hline i is a little more important than $j$ & $\tilde{3}=[2,3,4]$ \\
\hline $\mathrm{i}$ is more important than $\mathrm{j}$ & $\tilde{5}=[4,5,6]$ \\
\hline $\mathrm{i}$ is much more important than $\mathrm{j}$ & $\tilde{7}=[6,7,8]$ \\
\hline i is extremely more important than $\mathrm{j}$ & $\tilde{9}=[8,9,10]$ \\
\hline intermediate value of two neighboring judgments above & $\tilde{2}, \tilde{4}, \tilde{6} 、 \tilde{8}$ \\
\hline
\end{tabular}

This paper draws lessons from Lambda-Max and calculates fuzzy index weight of Fuzzy-AHP.

a)Let $\alpha=1$,calculate the decision matrix $T_{m}=\left(T_{i j m}\right)_{n \times n}$ with cut set $\alpha$ - and we get the weight with analytic hierarchy

process: $W_{m}=\left(W_{i m}\right)_{n \times n}, i=1,2, \cdots n$. 
b)Let $\alpha=0$,use cut set $\alpha$ and we obtain the upper and lower bounds decision matrix $T_{l}=\left(T_{i j l}\right)_{n \times n}, T_{n}=\left(T_{i j n}\right)_{n \times n}$, and we get the weight: $W_{l}=\left(W_{i l}\right)_{n \times n}, W_{n}=\left(W_{i n}\right)_{n \times n}, i=1,2, \cdots n$

c) weight adjustment

$$
\begin{aligned}
W_{l}^{*} & =\min \left(\frac{W_{i m}}{W_{i p}} 1 \leq i \leq n\right) *\left(W_{i l}\right)_{n \times n} \\
W_{n}^{*} & =\max \left(\frac{W_{i m}}{W_{i n}} 1 \leq i \leq n\right) *\left(W_{i l}\right)_{n \times n} \\
W_{i} & =\left(W_{i l}^{*}, W_{i m}, W_{i n}{ }^{*}\right)_{n \times n}
\end{aligned}
$$

d)fuzzy up the weight.

e)Checking consistency.

f)expertise aggregation

$$
W_{i-f}^{*}=\frac{1}{2}\left((1-\gamma) W_{i l}^{*}+W_{i m}+\gamma W_{i n}^{*}\right) \text { and among }
$$

that, $\gamma$ means investment preference degree

4) Determine the language weight decision-making matrix of risk indexes. The experts determine the language weight of photovoltaic building project based on the importance of indexes and establish judgment matrix of photovoltaic building project indexes showed in Table III

\section{TABLE III. JUDGMENT MATRIX}

\begin{tabular}{c|c|c|c|c}
\hline & $\boldsymbol{C}_{1}$ & $\boldsymbol{C}_{2}$ & $\ldots$ & $\boldsymbol{C}_{\boldsymbol{m}}$ \\
\hline $\mathbf{D}_{\mathbf{1}}$ & $\mathrm{W}_{11}$ & $\mathrm{~W}_{12}$ & $\ldots$ & $\mathrm{W}_{1 \mathrm{~m}}$ \\
\hline $\mathbf{D}_{\mathbf{2}}$ & $\mathrm{W}_{21}$ & $\mathrm{~W}_{22}$ & $\ldots$ & $\ldots$ \\
\hline$\ldots$ & $\ldots$ & $\ldots$ & $\ldots$ & $\ldots$ \\
\hline $\mathbf{D}_{\mathbf{k}}$ & $\mathrm{W}_{\mathrm{k} 1}$ & $\mathrm{~W}_{\mathrm{k} 2}$ & $\ldots$ & $\mathrm{W}_{\mathrm{km}}$ \\
\hline weight & $\mathrm{W}_{1}$ & $\mathrm{~W}_{2}$ & $\ldots$ & $\mathrm{W}_{\mathrm{m}}$ \\
\hline
\end{tabular}

Among that:

$$
\begin{array}{r}
w_{j}=\left[w_{1 j}, w_{2 j}, w_{3 j}\right]=\frac{1}{k} \sum_{i=1}^{k} w_{i j}, j=1,2, \cdots, m \\
w_{1 j}==\frac{1}{k} \sum_{i=1}^{k} w_{1 i j}, w_{2 j}==\frac{1}{k} \sum_{i=1}^{k} w_{2 i j}, w_{3 j}==\frac{1}{k} \sum_{i=1}^{k} w_{3 i j}
\end{array}
$$

5) Determine indexes' triangular fuzzy numbers judgment matrix. The triangular fuzzy numbers attribute value of photovoltaic building project risk evaluation factors which is showed in Table IV.

TABLE IV. TRIANGULAR FUZZY NUMBERS JUDGMENT MATRIX

\begin{tabular}{|c|c|c|c|c|c|}
\hline & & $C_{1}$ & $C_{2}$ & $\ldots$ & $C_{n}$ \\
\hline \multirow{3}{*}{$\mathbf{D}_{\mathbf{k}}$} & $\mathbf{A}_{1}$ & $\mathrm{X}_{11 \mathrm{k}}$ & $\mathrm{X}_{12 \mathrm{k}}$ & $\ldots$ & $\mathrm{X}_{1 \mathrm{mk}}$ \\
\cline { 2 - 6 } & $\ldots$ & $\ldots$ & $\ldots$ & $\ldots$ & $\ldots$ \\
\cline { 2 - 6 } & $\mathbf{A}_{\mathrm{m}}$ & $\mathrm{X}_{\mathrm{m} 1 \mathrm{k}}$ & $\mathrm{X}_{\mathrm{m} 2 \mathrm{k}}$ & $\ldots$ & $\mathrm{X}_{\mathrm{mmk}}$ \\
\hline
\end{tabular}

Among them:

$$
\begin{aligned}
& \mathrm{X}_{i j}=\left[o_{1 j}, p_{2 j}, q_{3 j}\right]=\frac{1}{k} \sum_{i=1}^{k} X_{i j}, i=1,2, \cdots, m, j=1,2, \cdots, m \\
& o_{i j}==\frac{1}{k} \sum_{i=1}^{k} o_{i j k}, p_{i j}==\frac{1}{k} \sum_{i=1}^{k} p_{i j k}, q_{i j}==\frac{1}{k} \sum_{i=1}^{k} w_{i j k}
\end{aligned}
$$

Total number of experts is $k$,the number of photovoltaic building project risk evaluation indexes is $m, X_{i j}$ is the weight of jth photovoltaic building project risk evaluation index determined by experts of kth sequence $(1 \leq j \leq m$ ).

6) Standardization of fuzzy judgment matrix the standardized fuzzy matrix of photovoltaic building project risk evaluation is:

$$
R=\left(r_{i j}\right)_{m \times n}, i=1,2, \cdots, m, j=1,2, \cdots, n
$$

Classify photovoltaic building project risk evaluation indexes into indexes of aggressive type and indexes of conservative type.let $\mathrm{B}$ be the index of aggressive type and let $\mathrm{C}$ be the index of conservative type,so calculation method of $\mathrm{r}_{\mathrm{ij}}$ is:

$$
\left\{\begin{array}{l}
r_{i j}=\left(\frac{a_{1 i j}}{a_{3 j}^{*}}, \frac{a_{2 i j}}{a_{3 j}^{*}}, \frac{a_{3 i j}}{a_{3 j}^{*}}\right), j \in B \\
r_{i j}=\left(\frac{a_{3 j}^{-}}{a_{3 i j}}, \frac{a_{3 j}^{-}}{a_{2 i j}}, \frac{a_{3 j}^{-}}{a_{1 i j}}\right), j \in C
\end{array}\right.
$$$$
\text { Among that, }\left\{\begin{array}{l}
a_{3 j}^{*}=\max _{i} a_{3 i j}, j \in B \\
a_{3 j}^{-}=\min _{i} a_{1 i j}, j \in C
\end{array}\right.
$$

7) Standardized weight decision-making matrix of photovoltaic building project risk evaluation:

$$
\begin{gathered}
V=\left(v_{i j}\right)_{m \times n} \\
v_{i j}=r_{i j} \otimes w_{i j}, i=1,2, \cdots, m, j=1,2, \cdots, n
\end{gathered}
$$

\section{8) Fuzzy up triangular fuzzy numbers}

Ambiguity resolution process of photovoltaic building project risk evaluation is:

a) triangular fuzzy numbers is as follows:

$$
f_{i j}=\left(l_{i j}, m_{i j}, r_{i j}\right), i=1,2, \cdots, m, j=1,2, \cdots, n
$$

b) Standardization is:

$$
\begin{aligned}
r_{i}^{\max } & =\max _{j} r_{i j}, l_{i}^{\min }=\min _{j} l_{i j} \\
\Delta_{\min }^{\max } & =\max _{j} r_{i j}-\min _{j} l_{i j}
\end{aligned}
$$

c) Then

$$
x_{l j}=\left(l_{i j}-l_{i}^{\min }\right) / \Delta_{\min }^{\max }
$$




$$
\begin{gathered}
x_{m j}=\left(m_{i j}-l_{i}^{\min }\right) / \Delta_{\min }^{\max } \\
x_{r j}=\left(r_{i j}-l_{i}^{\min }\right) / \Delta_{\min }^{\max }
\end{gathered}
$$

d) Calculate the left and right sides normalized values is :

$$
\begin{aligned}
& x_{j}^{l s}=x_{m j} /\left(1+x_{m j}-x_{r j}\right) \\
& x_{j}^{r s}=x_{r j} /\left(1+x_{r j}-x_{m j}\right)
\end{aligned}
$$

e) Calculating the total normalized crisp value is : $x_{j}^{\text {crisp }}=\left[x_{j}^{l s}\left(1-x_{j}^{l s}\right)+x_{j}^{r s} x_{j}^{r s}\right] /\left[1-x_{j}^{l s}+x_{j}^{r s}\right]$

f) the crisp value is:

$$
f_{i j}=l_{i}^{\min }+x_{j}^{\text {crisp }} \Delta_{\min }^{\max } .
$$

g) Calculate the total evaluation result

The ranking matrix $B=\left(B_{1}, B_{2}, B_{3}, \ldots, B_{m}\right)$ and the comprehensive evaluation result is $Q=B \circ V$.

\section{ANALYSIS OF AN EXAMPLE}

At first, according to evaluation indexes, decision-making experts establish linguistic assessment sets and evaluate the importance of various photovoltaic building project evaluation indexes which is showed in Table V. According to the results ,we determine the index weight showed in Table VI.

TABLE V. FUZZY EVALUATION VARIABLES OF RISK INDEX

\begin{tabular}{|c|c|c|}
\hline & construction equipment security(T34) & $(4.5,6.5,8.5)$ \\
\hline & job specification soundness(T41) & $(0.25,1.25,3)$ \\
\hline & operators'relative qualification(T42) & $(0.5,2,4)$ \\
\hline & technology support fault(T43) & $(0.5,2,4)$ \\
\hline & project management team(T51) & $(4.5,6.5,8.5)$ \\
\hline & management dispatch scheme(T52) & $(0.25,1.25,3)$ \\
\hline & information condition(T53) & $(6,7.75,9)$ \\
\hline & relay protection risk(T61) & $(0.25,1.25,3)$ \\
\hline & power reliability risk(T71) & $(7.5,9,9.75)$ \\
\hline & power stability risk(T81) & $(4.5,6.5,8.5)$ \\
\hline & power quality risk(T91) & $(7.5,9,9.75)$ \\
\hline \multirow{15}{*}{$\begin{array}{c}\text { economic } \\
\text { risks(E) }\end{array}$} & pool purchase price(E11) & $(7.5,9,9.75)$ \\
\hline & electricity to access grid(E21) & $(7,8.75,9.75)$ \\
\hline & operation cost risk(E31) & $(6.5,8.25,9.5)$ \\
\hline & extra cost risk(E41) & $(6.5,8.5,9.75)$ \\
\hline & efficiency risk(E51) & $(3,5,7)$ \\
\hline & operating profit ratio(E61) & $(6,7.75,9)$ \\
\hline & operating gross profit rate(E62) & $(7.5,9,9.75)$ \\
\hline & the profit margin of costs(E63) & $(7,8.75,9.75)$ \\
\hline & cash to current liability ratio(E71) & $(4.5,6.5,8.5)$ \\
\hline & equity ratio(E72) & $(3,5,7)$ \\
\hline & obtained interest multiple(E73) & $(7,8.75,9.75)$ \\
\hline & income growth rate(E81) & $(5.5,7.5,9.25)$ \\
\hline & net profit growth rate(E82) & $(7,8.75,9.75)$ \\
\hline & $\begin{array}{l}\text { accounts receivable turnover } \\
\text { ratio(E91) }\end{array}$ & $(0.5,2,4)$ \\
\hline & turnover rate of fixed assets(E92) & $(0.75,2.5,4.5)$ \\
\hline \multirow{5}{*}{$\begin{array}{c}\text { policy } \\
\text { risks(P) }\end{array}$} & investment allowance(P11) & $(3,5,7)$ \\
\hline & offset by tax revenue(P12) & $(0.25,1.25,3)$ \\
\hline & $\begin{array}{l}\text { purchasing electricity } \\
\text { compensation(P21) }\end{array}$ & $(7,8.75,9.75)$ \\
\hline & continuity index(P31) & $(8,9.25,9.75)$ \\
\hline & operability index(P41) & $(7.5,9,9.75)$ \\
\hline
\end{tabular}

\begin{tabular}{c|c}
\hline $\begin{array}{c}\text { risk evaluation indexes of photovoltaic building } \\
\text { project } \boldsymbol{C}\end{array}$ & fuzzy values \\
\hline poorer & $(0,1,3)$ \\
\hline poor & $(1,3,5)$ \\
\hline general & $(3,5,7)$ \\
\hline good & $(5,7,9)$ \\
\hline better & $(7,9,10)$ \\
\hline
\end{tabular}

TABLE VI. COMPREHENSIVE RISK EVALUATION INDEX SYSTEM

\begin{tabular}{|c|l|l|}
\hline risk & \multicolumn{1}{|c|}{ indexes } & evaluation sets \\
\hline \multirow{4}{*}{$\begin{array}{c}\text { natural } \\
\text { environment } \\
\text { risks(N) }\end{array}$} & latitude and longitude(N11) & $(3.5,5.5,7.5)$ \\
\cline { 2 - 3 } & sunshine duration(N12) & $(0.5,2,4)$ \\
\cline { 2 - 3 } & altitude and pressure(N13) & $(0.75,2.5,4.5)$ \\
\cline { 2 - 3 } & $\begin{array}{l}\text { air ground temperature and } \\
\text { humidity(N14) }\end{array}$ & $(0.75,2.5,4.5)$ \\
\cline { 2 - 3 } & geological hazard index(N21) & $(0.25,0.75,2)$ \\
\cline { 2 - 3 } & $\begin{array}{l}\text { comprehensive extreme climate } \\
\text { index(N22) }\end{array}$ & $(0.25,0.75,2)$ \\
\hline \multirow{5}{*}{$\begin{array}{l}\text { technology } \\
\text { risks(T) }\end{array}$} & $\begin{array}{l}\text { technology scheme design } \\
\text { feasibility(T11) }\end{array}$ & $(6,7.75,9)$ \\
\cline { 2 - 3 } & $\begin{array}{l}\text { commission design unit and designers } \\
\text { qualification(T12) }\end{array}$ & $(7,8.75,9.75)$ \\
\cline { 2 - 3 } & $\begin{array}{l}\text { Information and data } \\
\text { authenticity(T13) }\end{array}$ & $(3,5,7)$ \\
\cline { 2 - 3 } & design innovation(T14) & $(6,7.75,9)$ \\
\cline { 2 - 3 } & design applicability(T15) & $(7,8.75,9.75)$ \\
\cline { 2 - 3 } & equipment security(T21) & $(3,5,7)$ \\
\cline { 2 - 3 } & equipment stability(T22) & $(2,4,6)$ \\
\cline { 2 - 3 } & $\begin{array}{l}\text { equipment performance price } \\
\text { ratio(T23) }\end{array}$ & $(6,7.75,9)$ \\
\cline { 2 - 3 } & constructors' quality(T31) & $(5.5,7.5,9.25)$ \\
\cline { 2 - 3 } & personnel safety facility(T32) & $(5,6.75,8.25)$ \\
\cline { 2 - 3 } & $\begin{array}{l}\text { construction training and supervision } \\
\text { mechanism(T33) }\end{array}$ & $(7,8.75,9.75)$ \\
\hline
\end{tabular}

\begin{tabular}{|c|c|}
\hline risk dimensions & fuzzy evaluation sets \\
\hline $\begin{array}{ll}\begin{array}{l}\text { Natural } \\
\text { risk(N) }\end{array} & \text { environment } \\
\end{array}$ & {$[(0,0,0),(0.2,0.6,1),(1.5,2.5,3.5),(1.5,2.1,2.7),(0,0,0)]$} \\
\hline Technology risk(T) & {$[(0,0,0),(0.2,0.6,1),(1.8,3,4.2),(1,1.4,1.8),(0,0,0)]$} \\
\hline Economic risk(E) & {$[(0,0,0),(0.2,0.6,1),(1.5,2.5,3.5),(1.5,2.1,2.7),(0,0,0)]$} \\
\hline Policy risk(P) & {$[(0,0,0),(0.5,1.1,2.9),(1.8,2.8,3.5),(1,1.5,1.9),(0,0,0)]$} \\
\hline
\end{tabular}

We obtain the final fuzzy evaluating matrix as showed in Table VII

TABLE VII. COMPREHENSIVE RISK RESULTS

Evaluation result are as follows:

Fuzzification result:D=(0.7,2.46,7.51,6.19,0.67).

Fuzzy evaluation attributecan be written as follows:

$\mathrm{C}=($ lower,low,medium,high,higher $)=(50,60,70,80,90)$,so

$\mathrm{Q}=72.09$ belongs to medium risk.

\section{CONCLUSION}

By triangular fuzzy Number method, evaluation results are more scientific and reliable since some useful information is not wasted in the process of the evaluation. Risk factors of photovoltaic building project includes natural environment risks, technology risks, economic risks and policy risks. By triangular fuzzy Number method we can integrate all the index and sub index information, which makes the conclusion more credible.

\section{ACKNOWLEDGMENT}

The author thanks Project 12MS72 supported by "the Fundamental Research Funds for the Central Universities" for the financial support. 


\section{REFERENCES}

[1] Zhang Wenquan, Zhang Aijun, and Hu Qinghui, Study on the Theory and Method of Quantitative Risk Assessment of Power Companies, Electric Power Technologic Economics, vol. 3, pp. 52-56. 2007.

[2] Qu Jiao, Wang Yueting, and Qu Yanshen, A brief description of solar energy utilization on the promotion of building energy-saving emission reduction, China Building Materials, vol. 4, pp. 90-91, 2008.

[3] Yi Liu, Xu Zhang, Yongqiao An.Risk Assessment and Empirical Analysis of Grid connected Distributed Photovoltaic Power. APPEEC2010, 2010.3
[4] Yi Liu,XuZhang. Research of EMC based BIPV Project's B-S Pricing Model and Benefit Sharing Method. 2009 International Conference on Energy and Environment Technology, vol.2, pp.109-112. 2009.10

[5] Yi Liu, Xu Zhang. EMC Based Risk Asessment System and Empirical Analysis of BIPV Projects. 2009 International Conference on Energy and Environment Technology, vol.1, pp.113-116. 2009.10

[6] SUN Lu, LU Xiao, HAN Yi-na. Triangular Fuzzy Number Multi-target Group Decision Making Control and Instruments In Chemical Industry, vol. 37, pp. 73-77. 2010 